FERMILAB-Conf-03/379-A

\title{
Neutrino Mixing and Cosmology
}

\author{
Nicole F. Bell ${ }^{\mathrm{a} * \dagger}$ \\ a NASA/Fermilab Astrophysics Center, Fermi National Accelerator Laboratory, Batavia, Illinois \\ $60510-0500$
}

\begin{abstract}
We review the consequences of neutrino mixing in the early universe. For both active-sterile mixing or mixing between three active neutrinos only, the consequences of oscillations depend crucially upon the size of the universe's lepton number (relic neutrino asymmetry.)
\end{abstract}

\section{Introduction}

The relic neutrino background has never been directly detected, so we must resort to indirect means to infer its properties. One of the most useful tools available is Big Bang Nucleosynthesis (BBN). By putting neutrino oscillations together with BBN, we may shed light on neutrino mixing, cosmology, or both. We will outline the consequences of oscillations of the relic background neutrinos, for both active-active and active-sterile oscillation modes. The central issue we shall probe is: how big is the universe's lepton number?

While the baryon asymmetry of the universe is well determined, $n_{B} / n_{\gamma} \simeq 5 \times 10^{-10}$, the size of the lepton asymmetry is unknown. The simplest assumption is that the baryon and lepton asymmetries are of the same size, as would be the case if $B-L$ were conserved. However, there are viable models in which $L$ may be large while $B$ is small. Given constraints on charge neutrality, any large lepton asymmetry would have to be hidden in the neutrino sector.

Since neutrinos and antineutrinos should be in chemical equilibrium until they decouple at a temperature $T \sim 2 \mathrm{MeV}$, they may be welldescribed by Fermi-Dirac distributions with equal and opposite chemical potentials:

$$
f(p, \xi)=\frac{1}{1+\exp (p / T-\xi)},
$$

\footnotetext{
*nfb@fnal.gov

†Talk presented at TAUP 2003, Seattle, USA, 5-9 September 2003.
}

where $p$ denotes the neutrino momentum, $T$ the temperature, and $\xi$ the chemical potential in units of $T$. The lepton asymmetry $L_{\alpha}$ for a given flavour $\nu_{\alpha}$ is related to the chemical potential by

$$
L_{\alpha}=\frac{n_{\nu_{\alpha}}-n_{\bar{\nu}_{\alpha}}}{n_{\gamma}}=\frac{\pi^{2}}{12 \zeta(3)}\left(\xi_{\alpha}+\frac{\xi_{\alpha}^{3}}{\pi^{2}}\right)
$$

where $\zeta(3) \simeq 1.202$. A nonzero chemical potential results in extra energy density, such that the effective number of neutrinos is increased from the standard model prediction by

$$
\Delta N_{\nu}=\frac{30}{7}\left(\frac{\xi}{\pi}\right)^{2}+\frac{15}{7}\left(\frac{\xi}{\pi}\right)^{4} .
$$

Large chemical potentials affect BBN in two ways:

1. The extra energy density increases the expansion rate of the universe, thus increasing the BBN helium abundance, and also alters the CMB results. This sets the weak bound $\left|\xi_{\alpha}\right| \lesssim 3$, for all three flavours.

2. An additional, much stronger, limit can be placed on the $\nu_{e}-\bar{\nu}_{e}$ asymmetry, as it directly effects the neutron to proton ratio prior to BBN by altering beta-equilibrium. (Beta-equilibrium is between the weak interactions $n+\nu_{e} \leftrightarrow p+e^{-}$and $p+\bar{\nu}_{e} \leftrightarrow$ $n+e^{+}$.) For example, positive $\xi_{e}$ increases the $\nu_{e}$ abundance relative to $\bar{\nu}_{e}$, thus lowering the neutron to proton ratio and decreasing the helium yield. This sets the limit $\left|\xi_{e}\right| \lesssim 0.04$. 
However, it is possible that the two effect compensate for each other, i.e., the effects of a small $\xi_{e}$ are partially undone by an increased expansion rate due to a large $\xi_{\mu, \tau}$. In this case the bounds become 1]:

$$
\begin{aligned}
-0.01<\xi_{e} & <0.22, \\
\left|\xi_{\mu, \tau}\right| & <2.6,
\end{aligned}
$$

where the upper limits are obtained only in tandem.

\section{Oscillations between three active neu- trino}

Since we know neutrinos oscillate, the individual lepton numbers $L_{e}, L_{\mu}$ and $L_{\tau}$ are violated and only the total lepton number is conserved. It was suggested in Ref. 2] (see also [3]) that the large neutrino mixing angles implied by the present data may lead to equilibration of all three flavours in the early universe. If a large asymmetry hidden in $\xi_{\mu, \tau}$ were to be transfered to $\xi_{e}$ well before weak freezeout at $T \simeq 1 \mathrm{MeV}$, the stringent BBN limit on $\xi_{e}$ would then apply to all three flavours, improving the bounds on $\xi_{\mu, \tau}$ by nearly two orders of magnitude.

This proposal was recently studied in detail in Refs. 4516, where close to complete equilibration of the asymmetries $\xi_{\mu, \tau}$ with $\xi_{e}$ was found. The equilibration takes place when $T \sim 2 \mathrm{MeV}$ via an MSW transition which is driven by the solar mass squared difference. (It is actually more complicated than a normal MSW transition, as forward scattering from other neutrinos in the background medium introduces a non-linear contribution to the effective potential [7]. This has the effect of synchronizing the neutrino ensemble such that all momentum modes go through the MSW resonance together, with parameters governed by an effective momentum, which is close to the thermal average. See 4568 for details.) This MSW transition converts the initial flavour states (which are approximately mass eigenstates at high temperature) into vacuum mass eigenstates, which, due to the large solar mixing angle, have large components of all three flavours.

This equilibration is an important result, as it excludes the possibility of degenerate BBN [9], and is the strongest limit on the total lepton number of the universe and is likely to remain so for the foreseeable future. In terms of extra relativistic degrees of freedom, the limit is impressively tight: If $\xi_{e, \mu, \tau}=0.04$, then $\Delta N_{\nu} \simeq 3 \times 0.0007=$ 0.002. One implication is that cosmological constraints on (and future measurements of) neutrino masses will not be subject to uncertainty in the relic neutrino density.

Strictly speaking, one version of the degenerate BBN scenario is still possible: It is conceivable that $\xi_{e} \sim \xi_{\mu} \sim \xi_{\tau} \sim 0.2$, provided that another relativistic particle species contributes the extra energy density required to compensate for the large $\nu_{e}$ chemical potential [510]. This extra energy density can no longer consist of active neutrino, so would have to be something more exotic. Such an unnatural scenario could eventually be detected via the CMB.

\section{Active-sterile oscillations}

Active-sterile oscillations before the time of BBN are potentially dangerous, as they may bring the sterile degrees of freedom into thermal equilibrium, thus increasing the expansion rate and thereby upsetting the successful predictions of BBN. All models which seek to explain LSND 11 via the addition of a sterile neutrino suffer from this problem. Since the LSND mixing angle is relatively large, the sterile neutrino is always thermalised [12].

The rate at which the sterile species is populated is

$$
\Gamma\left(\nu_{\alpha} \rightarrow \nu_{s}\right) \simeq \frac{1}{2} \Gamma_{\text {scatt }} \sin ^{2} 2 \theta_{m},
$$

where $\Gamma_{\text {scatt }}$ is he active-neutrino collision rate, and $\theta_{m}$ is the matter affected mixing angle. However, it is possible to avoid thermalisation of the sterile, by invoking the presence of a small relic neutrino asymmetry [13. This provides an index of refraction, which suppresses the active-sterile mixing angle so that the rate of sterile production is negligible, until after the neutrinos thermally decouple from the rest of the plasma. 


\section{Cosmological neutrino mass limits, and 4-neutrino models}

The absolute neutrino mass scale may be probed via cosmological means through large scale structure measurements, as free streaming of neutrinos suppresses the growth of structure on the small scales that are within the horizon while the neutrinos are relativistic. The current limit is roughly $\sum m_{\nu} \lesssim 1 \mathrm{eV}$, depending somewhat on how conservatively parameter degeneracies are priors are treated [14.

Since the LSND mass squared difference is $\sim O(\mathrm{eV})$, if all 4 neutrino species are populated in the universe, the cosmological mass limits constrain the LSND parameter space. However, BBN considerations already disfavour scenarios in which a 4 th neutrino is populated. If we avoid the thermalisation of the sterile state (via the presence of a lepton asymmetry) it may have two desirable consequences: In addition to eliminating the BBN problems, the large scale structure mass limits may also be avoided. For instance, if we have a $3+1$ model in which the heaviest, isolated, mass state consists mostly of the sterile neutrino, the abundance of this heavy state (and hence its contribution to $\Omega_{\nu}$ ) will be small.

\section{Conclusions}

Large angle MSW transitions lead to neutrino flavour equilibration in the early universe. This sets the strongest limit on the universe's lepton number, because stringent constraints on the $\nu_{e}$ $-\bar{\nu}_{e}$ asymmetry can now be applied to all three flavours. The possibility of "degenerate" BBN is thus eliminated, thereby removing a possible uncertainty in cosmological determinations of neutrino mass.

Sterile neutrino are cosmologically disfavoured if they are brought into thermal equilibrium before BBN. While all sterile neutrino models which can accommodate LSND suffer this problem, the constraints can be avoided by the presence of a lepton asymmetry, which prevents thermalisation of the sterile, thus avoiding both BBN limits and large scale structure mass limits.
Acknowledgments - N.F.B. was supported by Fermilab (operated by URA under DOE contract DE-AC02-76CH03000) and by NASA grant NAG5-10842.

\section{REFERENCES}

1. J. P. Kneller, R. J. Scherrer, G. Steigman and T. P. Walker, Phys. Rev. D 64, 123506 (2001); S. H. Hansen, G. Mangano, A. Melchiorri, G. Miele and O. Pisanti, Phys. Rev. D 65, 023511 (2002).

2. C. Lunardini and A. Y. Smirnov, Phys. Rev. D 64, 073006 (2001).

3. M. J. Savage, R. A. Malaney and G. M. Fuller, Astrophys. J. 3681 (1991).

4. A. D. Dolgov S. H. Hansen, S. Pastor, S. T. Petcov, G. G. Raffelt and D. V. Semikoz, Nucl. Phys. B 632, 363 (2002).

5. K. N. Abazajian, J. F. Beacom and N. F. Bell, Phys. Rev. D 66, 013008 (2002).

6. Y. Y. Wong, Phys. Rev. D 66, 025015 (2002).

7. J. Pantaleone, Phys. Lett. B 287, 128 (1992); A. Friedland and C. Lunardini, Phys. Rev. D 68, 013007 (2003); JHEP 0310, 043 (2003); N. F. Bell, A. A. Rawlinson and R. F. Sawyer, Phys. Lett. B 573, 86 (2003).

8. S. Pastor, G. G. Raffelt and D. V. Semikoz, Phys. Rev. D 65, 053011 (2002).

9. H. S. Kang and G. Steigman, Nucl. Phys. B 372, 494 (1992); S. Esposito, G. Mangano, G. Miele and O. Pisanti, JHEP 0009, 038 (2000); M. Orito, T. Kajino, G. J. Mathews and Y. Wang, Phys. Rev. D 65, 123504 (2002).

10. V. Barger, J. P. Kneller, P. Langacker, D. Marfatia and G. Steigman, Phys. Lett. B 569, 123 (2003); J. P. Kneller, talk presented at TAUP 2003.

11. A. Aguilar et al., Phys. Rev. D 64, 112007 (2001).

12. P. Di Bari, Phys. Rev. D 65, 043509 (2002) [Addendum-ibid. D 67, 127301 (2003)]; K. N. Abazajian, Astropart. Phys. 19, 303 (2003).

13. R. Foot and R. R. Volkas, Phys. Rev. Lett. 75, 4350 (1995).

14. S. Hannestad, JCAP 0305, 004 (2003). 